\title{
The structure of Mycobacterium tuberculosis heme-degrading protein, MhuD, in complex with product
}

Alex Chao ${ }^{1 \ddagger}$, Kalistyn H. Burley ${ }^{2 \ddagger}$, Paul J. Sieminski ${ }^{1}$, David L. Mobley ${ }^{2,3}$ and Celia W. Goulding ${ }^{1,2 *}$

AUTHOR ADDRESS ${ }^{1}$ Department of Molecular Biology \& Biochemistry, ${ }^{2}$ Pharmaceutical Sciences, \& ${ }^{3}$ Chemistry, University of California Irvine, Irvine, CA 92697

KEYWORDS. Heme degradation, Mycobacterium tuberculosis, mycobilin, biliverdin, iron

${ }^{\ddagger}$ Contributed Equally

\section{Corresponding Author}

*E-mail: celia.goulding@uci.edu

\section{ORCID}

Celia W. Goulding: 0000-0001-5582-0565

Alex Chao: 0000-0003-2134-1632

Kalistyn H. Burley: 0000-0002-7885-1812

David L. Mobley: 0000-0002-1083-5533

Paul J. Sieminski: 0000-0002-8467-5800 


\begin{abstract}
Mycobacterium tuberculosis (Mtb), the causative agent of tuberculosis, requires iron for survival. In Mtb, MhuD is the cytosolic protein that degrades imported heme. MhuD is distinct, both in sequence and structure, from canonical heme oxygenases (HOs) but homologous with IsdG-type proteins. Canonical HO is found mainly in eukaryotes, while IsdG-type proteins are predominantly found in prokaryotes including pathogens. While there are several published structures of MhuD and other IsdG-type proteins in complex with heme substrate, no structures have been reported of IsdG-type proteins in complex with product, unlike HOs. We recently showed that the Mtb variant MhuD-R26S produces biliverdin IX $\alpha(\alpha B V)$ rather than the wild-type (WT) mycobilin isomers as product. Given that mycobilin and other IsdG-type protein products like staphylobilin are difficult to isolate in quantities sufficient for structure determination, here we use the MhuD-R26S variant and its product $\alpha \mathrm{BV}$ as a proxy to study the IsdG-type protein/product complex. First we show that $\alpha B V$ has nanomolar affinity for MhuD and the R26S variant. Second we determined the MhuD-R26S- $\alpha$ BV complex structure to $2.5 \AA$, which reveals two notable features (1) two $\alpha \mathrm{BV}$ molecules bound per active site and (2) a new $\alpha$-helix ( $\alpha 3$ ) as compared with the MhuD-heme structure. Finally, by molecular dynamics simulations we show that $\alpha 3$ is stable with the proximal $\alpha B V$ alone. MhuD's high affinity for its product and structural and electrostatic changes that accompany substrate turnover suggest that there is an unidentified protein that is responsible for product extraction from MhuD and other IsdG-type proteins.
\end{abstract}




\section{Introduction}

Heme degradation is important for a variety of biological functions, including iron reutilization, cell signaling, and antioxidant defense (Brouard et al., 2000; Dore et al., 1999; Ferris et al., 1999). The well-studied canonical heme oxygenase (HO), human $\mathrm{HO}(\mathrm{hHO}-$ 1), catalyzes the oxidative cleavage of heme to release biliverdin $\mathrm{Xl} \alpha$ ( $\alpha \mathrm{BV}$, Figure 1A), ferrous iron, and carbon monoxide (CO) (Matsui et al., 2010; Tenhunen et al., 1969; Yoshida et al., 1980). HO homologs have also been found in many eukaryotes and some prokaryotes including the pathogens, Corynebacterium diphtheriae, Pseudomonas aeruginosa and Neisseria meningitidis, and predominately produce the same heme degradation products (Ratliff et al., 2001; Schmitt, 1997; Wilks, 2002; Wilks and Schmitt, 1998; Zhu et al., 2000). In eukaryotes, the HO reaction is coupled with the conversion of biliverdin (BV) to bilirubin by biliverdin reductase (BVR) (Noguchi et al., 1979). After conjugation of bilirubin with glucuronic acid, bilirubin is excreted (Mantle, 2002). The fate of HO-produced BV in prokaryotes has not been well studied thus far. Although in $P$. aeruginosa, it has been shown that extracellular acquired heme is degraded by a $\mathrm{HO}$ homolog, HemO, whereby the BV by-product is then excreted, but not further reduced, by an unknown mechanism (Barker et al., 2012).

There is a heme-degrading protein family distinct, both in sequence and structure, from canonical HOs mainly found in bacteria, and termed the iron surface determinant $G$ (IsdG)-type family (Chim et al., 2010; Skaar et al., 2004; Wu et al., 2005). Staphylococcus aureus IsdG and Isdl were the first members characterized (Skaar et al., 2004; Wu et al., 2005), and instead of degrading heme to BV, iron and CO, these enzymes cleave and oxidize heme at the $\beta$ - and $\delta$-meso carbon sites to produce staphylobilin isomers (Figure 1A), free iron and formaldehyde (Matsui et al., 2013; Reniere et al., 2010). Other IsdGtype enzymes have been identified including MhuD from Mycobacterium tuberculosis (Mtb) and LFO1 from eukaryotic Chlamydomonas reinhardtii, and both degrade heme into unique products (Chim et al., 2010; Lojek et al., 2017). While LFO1 degrades heme into a yet-to-be-determined product(s) (Lojek et al., 2017), MhuD has been shown to degrade heme into iron and mycobilin isomers (Figure 1A) (Nambu et al., 2013). Like 
staphylobilins, the mycobilin isomers are also oxidized at the $\beta$ - or $\delta$-meso carbons; however cleavage occurs at the a-meso carbon with no observed loss of a C1-product (Nambu et al., 2013). It was proposed that there is no C1-product as $\mathrm{hHO}-1$ produced CO triggers the transition of Mtb from its active to latent state (Nambu et al., 2013). The fate of the IsdG-type protein heme degradation tetrapyrrole products, like staphylobilin and mycobilin, is unknown; however they may have antioxidant properties similar to BV (Vanella et al., 2016).

The structures of $\mathrm{HO}$ and IsdG-type proteins are quite distinct. HOs are comprised of a monomeric a-helical domain (Schuller et al., 1999), while IsdG-type proteins consist of a dimeric $\beta$-barrel decorated with two $\alpha$-helices from each monomer (Wu et al., 2005). Unsurprisingly, the two distinct classes of heme-degrading enzymes degrade heme by different mechanisms (Matsui et al., 2016). Although heme is coordinated by a proximal His residue in both $\mathrm{HO}$ and IsdG-type enzymes, the heme molecule in $\mathrm{HOs}$ is near-planar, while for IsdG-type proteins, the heme is ruffled (Schuller et al., 1999; Wu et al., 2005). Furthermore, $\mathrm{HO}$ has a distal pocket with a network of ordered water molecules that facilitates the three consecutive monooxygenase steps required for heme degradation (Matsui et al., 2010). In contrast, the distal heme pocket is quite hydrophobic for IsdGtype proteins, with only one or two ordered waters observed in the proximal pocket (Graves et al., 2014; Wu et al., 2005). In MhuD, it has been proposed that the hydrophobic pocket together with the ruffled heme contributes to the sequential monooxygenase and dioxygenase steps necessary for MhuD to degrade heme (Matsui et al., 2016).

The structures of both human and $C$. diphtheriae $\mathrm{HO}$ complexed with $\alpha \mathrm{BV}$ illustrate that the $\mathrm{HO}$ heme degradation reaction is coupled with a conformational change from a 'closed 'to 'open' state (Lad et al., 2004; Unno et al., 2013). In both the eukaryotic and prokaryotic $\mathrm{HO}$ structures, the open-product bound state results from relaxation of the distal and proximal helices, and a rotation of the catalytic His side-chain out of the active site pocket as it is no longer coordinated to heme-iron (Lad et al., 2004; Unno et al., 2013). This structural shift suggests that a degree of protein flexibility is necessary amongst the $\mathrm{HO}$ homologs to facilitate catalysis. Structures of apo and monoheme bound forms of IsdG- 
type proteins reveal an analogous conformational change in the catalytic His residue as it is absent or disordered in the apo structures; however the ordering of the elongated L2 loop region upon heme binding is a much more drastic conformational shift as compared with HOs (apo-MhuD; Protein Data Bank (PDB) ID: 5UQ4) (Graves et al., 2014; Lee et al., 2008; Wu et al., 2005). Compared with other studied IsdG-type proteins, the MhuD active site is exceptionally flexible and can accommodate two molecules of heme, resulting in protein inactivation (Chim et al., 2010; Graves et al., 2014). The biological significance of this conformational plasticity and its role in product turnover is not well understood as there is no structure of an IsdG-type enzyme in its product-bound form.

The structure of an IsdG-type protein in complex with its heme degradation product would further our understanding of the mechanism of action of this protein family. Staphylobilin and mycobilin products of IsdG-type proteins are difficult to purify (Nambu et al., 2013; Reniere et al., 2010), which has presumably hindered the structural analysis of the product-bound form. Recently, we demonstrated that a MhuD variant, MhuD-Arg26Ser (Figure 1B), upon heme degradation produces $\alpha \mathrm{BV}$, formaldehyde and iron (Chao and Goulding, 2019). In this current study, we determine the affinity of MhuD and the MhuDR26S variant to both heme and $\alpha B V$, and show they both bind heme and $\alpha B V$ in the nanomolar range. This high affinity to $\alpha B V$ has allowed us to utilize the MhuD-R26S variant as a proxy to study the IsdG-type proteins in complex with product. Upon solving the crystal structure of the MhuD-R26S- $\alpha$ BV, we observed the formation of a novel secondary structural element as compared to the heme-bound MhuD structure, and its implications will be discussed further.

\section{Methods}

Fluorescence-detection of ligand binding

Fluorescence-detected titrations of heme and $\alpha \mathrm{BV}$ were carried out using a protocol previously described (Thakuri et al., 2018). Stock solutions of MhuD (80 nM), heme (8 $\mu \mathrm{M})$, and $\alpha \mathrm{BV}(8 \mu \mathrm{M})$ were prepared in $50 \mathrm{mM}$ Tris/ $\mathrm{HCl} \mathrm{pH} 7.4,150 \mathrm{mM} \mathrm{NaCl}$. Heme or aBV was titrated and gently mixed in $16 \mathrm{nM}$ or $32 \mathrm{nM}$ increments into MhuD-WT or MhuDR26S. Following a 1-min incubation, fluorescence emission spectra were acquired 
between 320 to $500 \mathrm{~nm}$ on a Hitachi F-4500 Fluorescence Spectrophotometer through excitation at $285 \mathrm{~nm}$ with the following parameters: $1 / 3 \mathrm{~nm}$ step size, scan speed of 240 $\mathrm{nm} / \mathrm{min}$, PMT voltage of $700 \mathrm{~V}$, and slit widths at $10 \mathrm{~nm}$ (MhuD WT) and $20 \mathrm{~nm}$ (MhuDR26S).

Fluorescence emission spectral analysis

Results from the above fluorescence-based assay were fitted to Eqn. 1 derived from Conger et el (Conger et al., 2017; Thakuri et al., 2018), to determine the equilibrium dissociation-constant $\left(K_{d}\right)$ of heme or $\mathrm{\alpha BV}$ with MhuD and its mutants.

Eqn. 1:

$$
\begin{aligned}
F=\frac{\left([\text { MhuD }]+[\text { ligand }]+K_{d}\right)-\sqrt{\left([\text { MhuD }]+[\text { ligand }]+K_{d}\right)^{2}-4[\text { MhuD }][\text { Heme }]}}{2} \\
\times\left(\frac{F_{\min }-F_{\max }}{[\text { MhuD }]}\right)+F_{\max }
\end{aligned}
$$

In Eqn. 1, [MhuD] is the total concentration of MhuD or mutant MhuD, [ligand] is the total concentration of heme or $\alpha B V, F_{\max }$ is the emission intensity with ligand absent, and $F_{\min }$ is the emission intensity for fully ligand-bound MhuD. Fitting of the fluorescence emission intensity at $340 \mathrm{~nm}$ for $K_{d}$ determination was performed using Origin 2018.

\section{Expression and purification of Mtb MhuD and the R26S variant}

Wild-type (WT) MhuD and the R26S variant were purified as previously reported (Chao and Goulding, 2019; Chim et al., 2010). In brief, E. coli B21-Gold (DE3) cells transformed with pET22b-MhuD plasmid were grown in LB medium containing $50 \mu \mathrm{g} / \mathrm{mL}$ ampicillin at $37^{\circ} \mathrm{C}$. Overexpression was induced at $\mathrm{OD}_{600}$ of $\sim 0.6$ using $1 \mathrm{mM}$ IPTG. The cells were harvested 4 hours post induction and resuspended in lysis buffer $(50 \mathrm{mM}$ Tris/ $\mathrm{HCl}$ pH 7.4, $350 \mathrm{mM} \mathrm{NaCl}$ and $10 \mathrm{mM}$ imidazole). Cells were lysed via sonication and the resulting lysate was centrifuged at $14,000 \mathrm{rpm}$. The cell supernatant was loaded onto a $\mathrm{Ni}^{2+}$ charged HiTrap chelating column $(5 \mathrm{~mL})$ and washed with lysis buffer. Bound protein was eluted from the column with increasing concentrations of imidazole. Next, MhuD, which elutes at 50 and $100 \mathrm{mM}$ imidazole, was concentrated (Amicon, $5 \mathrm{kDa}$ molecular mass 
cutoff) and was further purified on a $\mathrm{S} 75 \mathrm{gel}$ filtration column in $20 \mathrm{mM}$ Tris/ $\mathrm{HCl} \mathrm{pH} 8$, and $10 \mathrm{mM} \mathrm{NaCl}$.

\section{Crystallization of MhuD-R26S- $\alpha B V$ complex}

To prepare an $\alpha B V$ solution, approximately $2 \mathrm{mg}$ of aBV hydrochloride (SigmaAldrich) was dissolved in $500 \mu \mathrm{L} 0.1 \mathrm{M} \mathrm{NaOH}$ followed by $500 \mu \mathrm{L} 1 \mathrm{M}$ Tris/HCl pH 7.4 before dilution into $50 \mathrm{mM}$ Tris/ $\mathrm{HCl}$ pH 7.4, $150 \mathrm{mM} \mathrm{NaCl}$. A ferric chloride solution was prepared by dissolving $27.3 \mathrm{mg}$ of ferric chloride hexahydrate (SigmaAldrich) in $10 \mathrm{~mL}$ water. A 1.3 fold excess of a 1:1 molar ratio solution of $\alpha B V$ and ferric chloride was gradually added to $100 \mu \mathrm{M}$ apo-MhuD R26S and incubated overnight at $4^{\circ} \mathrm{C}$ before being concentrated to $10 \mathrm{mg} / \mathrm{mL}$ (Lowry assay (Lowry et al., 1951)). Light blue crystals appeared in $0.1 \mathrm{M}$ HEPES pH 6.5, 4.6 M NaCl, 30 mM glycyl-glycyl-glycine after 2 days. The crystals were flash frozen in $100 \% \mathrm{NVH}$ oil and a data set to $2.5 \AA$ was collected at $70 \mathrm{~K}$. The collected data was indexed, integrated, and reduced using iMOSFLM (Battye et al., 2011). Initial phase determination was carried out using Phaser in the PHENIX suite (Adams et al., 2010) using WT MhuD-heme-CN structure as a search model (PDB ID 4NL5) (Graves et al., 2014). aBV molecules were positioned into appropriate positive electron density in the vicinity of the active site, and the structure was refined using phenix.refine (Adams et al., 2010). For each promoter, the electron density of the loop region between Ala24 to Asn32 is poorly defined and residues His25-Val30 were modeled as alanines. The only Ramanchandran outliers are Val30 modeled as Ala in both Chain A and B, which are in this poorly defined region of electron density.

\section{Molecular dynamics (MD) simulations}

The input files for $\mathrm{MhuD}^{4 N L 5}$-heme and $\mathrm{MhuD}^{4 N L 5}-\alpha \mathrm{BV}$ simulations were prepared from the structure of dimeric MhuD-heme-CN and MhuD ${ }^{4 N L 5}$-heme did not include the cyano group (PDB ID: 4NL5). For the MhuD- $\alpha$ BV complex, input files were prepared from the MhuD-R26S- $\alpha$ BV structure, wherein the R26S mutation was reversed and just one $\alpha B V$ (proximal) was retained per active site. All crystallographic waters were removed with the exception of ordered waters in the active site of MhuD ${ }^{4 N L 5}$ (HOH numbers: $313,325,349$ ). Hydrogen atoms were added to the system using pdb4amber from AmberTools15 with 


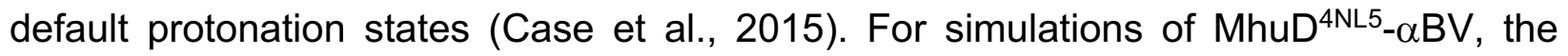
coordinates of heme atoms served as a scaffold upon which each $\alpha B V$ was manually docked. $\alpha B V$ was parameterized using antechamber from AmberTools15 (Case et al., 2015) with GAFF version 1.7 and AM1-BCC charges. The ligated His75-Fe-heme ligand was parameterized according to previously published Density Functional Theory calculations (Harris et al., 2001). Missing atoms for each structure were added using tleap in AmberTools15 (Case et al., 2015), and parameterized using ff99SBildn (LindorffLarsen et al., 2010). Each system was explicitly solvated in tleap with a $10 \AA$ rectangular box of TIP3P water, extending from the surface of the protein to the box edge, and sodium ions were added to neutralize the charge of the system.

Minimization proceeded using sander from Amber14 (Case et al., 2015) with steepest descents running for 20,000 timesteps of 2 fs each, followed by heating from $100 \mathrm{~K}$ to $300 \mathrm{~K}$ with constant volume for 25,000 timesteps of $2 \mathrm{fs}$. Equilibration continued using sander for 500,000 timesteps of 2 fs under constant pressure with positional restraints initially applied on all non-water atoms at $50 \mathrm{kcal} / \mathrm{mol} / \AA^{2}$ and progressively lifted in increments of $5 \mathrm{kcal} / \mathrm{mol} / \AA^{2}$ over ten 50,000 step segments. The resulting topology and coordinate files for each system were used as inputs for MD simulations. Production simulations were executed in OpenMM 7.1.0 (Eastman et al., 2017) using a Langevin integrator with a 2 fs timestep and a friction coefficient of 10/ps. For each of the three systems, five independent simulations of 100 ns each were initiated with randomized velocities. Among the five simulations, one simulation was restarted for each system for an additional $500 \mathrm{~ns}$, bringing the total simulation time to $1 \mu \mathrm{s} / \mathrm{system}$.

\section{MD analysis}

Distances and secondary structure assignments were computed using MDTraj (McGibbon et al., 2015). For analysis, each monomer of MhuD was treated independently with the assumption that long-range interactions between each subunit are negligible for the time scales simulated here. To analyze the orientation of His75 during simulations, two orientations were defined: 1) Active Site - side chain pointing into binding pocket, towards the center of the tetrapyrrole, and 2) Solvent Exposed - side chain flipped away 
from the binding pocket, as in the MhuD- $\alpha B V$ structure (see Figure 4Bi). To assign these positions, we computed the distance between the $\varepsilon$ nitrogen atom (furthest from the backbone) on His75 and the nitrogen atom located between the $\alpha$ and $\beta$ carbons on either $\alpha B V$ or heme. If the distance was less than $6 \AA$, the His75 residue was classified as being oriented in the Active Site position; otherwise the position was classified as Solvent Exposed.

For the Arg79 positional analysis, we identified four positions; 1) Active site - Arg79 directed into the binding pocket interacting with the ligand, 2) Helix 1 - Arg79 side-chain interacting with residues on $\alpha$-helix-1 (i.e residues 16-25) of MhuD, 3) Helix 2 - Arg79 side-chain interacting with residues on $\alpha$-helix-2 (residues 60-75), and 4) Solvent Exposed - Arg79 side-chain oriented into the surrounding solvent. To analyze the Arg79 in our simulations, position assignments were defined as follows: 1) Active Site guanidinium carbon of Arg79 side-chain (CZ) within $4.5 \AA$ of either terminal carbon on the propionate groups of the heme or $\alpha \mathrm{BV}$ ligands. 2) Helix 1 - Arg79 CZ atom within $6 \AA$ of Glu16 $\alpha$ carbon 3) Helix 2 - Arg79 CZ atom within 6 Å of His75 or lle72 backbone oxygen and more than $4.5 \AA$ from terminal carbons on the propionate groups of the heme or $\alpha B V$ ligands. 4) Solvent Exposed - Any position of Arg79 falling outside the description of positions 1-3.

\section{Results}

\section{Affinity of substrate and product to MhuD}

Previously, the affinity $\left(K_{d}\right)$ of WT MhuD for heme was measured to be $\sim 6 \mathrm{nM}$ (Thakuri et al., 2018); empirically it has been also been observed that MhuD also has a high affinity for its product, as isolation of MhuD mycobilin products requires an iron-chelator followed by protein denaturation (Chao and Goulding, 2019; Nambu et al., 2013). Recently, we demonstrated that the MhuD-R26S variant degrades heme to produce $\alpha B V$ as its predominate tetrapyrrole product rather than the WT MhuD mycobilin isomers (Chao and Goulding, 2019; Nambu et al., 2013). As it is difficult to isolate large quantities of mycobilin, we use the MhuD-R26S variant and its $\alpha \mathrm{BV}$ product as a model system to 
study IsdG-type proteins in complex with product. First, we determined the affinities of heme and $\alpha B V$ to both WT MhuD and the R26S variant. The heme affinity was measured using a previously described fluorescence-based assay (Figure 2) (Thakuri et al., 2018). The $K_{d}$ of heme for MhuD-WT and MhuD-R26S are similar, both about 6 nM (Table 1), whereas the $K_{d}$ values of $\alpha B V$ for MhuD-WT and MhuD-R26S are $\sim 36 \mathrm{nM}$ and $\sim 92 \mathrm{nM}$, respectively (Table 1). The fact that WT MhuD binds $\alpha \mathrm{BV}$ with a higher affinity than the R26S variant (even though the product of WT MhuD is mycobilin rather than $\alpha B V$ ) implies that the Arg26 residue may form contacts with the tetrapyrrole product.

\section{The structure of the MhuD-R26S- $\alpha$ BV complex}

To investigate the structural impacts on MhuD arising from heme degradation into product, we turned our attention to structure determination of the MhuD-R26S variant in complex with $\alpha \mathrm{BV}$. The MhuD-R26S- $\alpha \mathrm{BV}$ complex crystallized in the presence of ferric chloride, and light blue crystals appeared in 0.1 HEPES pH 6.5, 4.6 M NaCl and $30 \mathrm{mM}$ glycyl-glycyl-glycine after 2 days. The structure of the MhuD-R26S-aBV complex was solved to $2.5 \AA$ resolution with a final $R / R_{\text {free }}$ of $23.2 / 28.5$ (Table 2 ). The asymmetric unit contains two MhuD-R26S promoters bridged between their active sites by five partially $\pi-$ stacked aBV molecules (Figure 3A). The MhuD-R26S monomers each complexed with two $\alpha B V$ molecules superimpose with a root-mean-square deviation (RMSD) of $0.2 \AA$. The biologically relevant MhuD-R26S homodimer is observed in the crystallographic 2fold symmetry (Figure 3B), similar to the apo- and heme-bound MhuD structures (apoMhuD; PDB code: 5UQ4) (Chim et al., 2010; Graves et al., 2014).

The MhuD-R26S-aBV structure is similar to that of the MhuD-heme-CN and MhuDdiheme structures (Chim et al., 2010; Graves et al., 2014), where each subunit forms a ferredoxin-like fold. Two monomers form a dimeric antiparallel $\beta$-barrel where each monomer has three $\alpha$-helices together with two flexible loop regions; the first loop connects $\alpha$-helix-1 to $\beta$-strand- 2 and the second connects $\alpha$-helix-3 to $\beta$-strand-4 (Figure 3B). It should be noted that the topology of the MhuD-R26S- $\alpha$ BV complex differs from the structures of apo-MhuD and heme-bound WT-MhuD (Graves et al., 2014). Within the MhuD-R26S- $\alpha$ BV structure, there is an additional short $\alpha$-helix-3 formed by residues 
Ala76-Asn81, which was not observed in the monoheme, diheme and apo-MhuD structures (Chim et al., 2010; Graves et al., 2014), and thus the latter structures have a slightly longer extended L2 loop region. There are also other subtle conformational changes that will be discussed later.

\section{aBV Binding to MhuD}

Each MhuD-R26S active site binds two molecules of aBV (Figures 3B). The solventprotected or proximal $\alpha \mathrm{BV}$ interacts with both MhuD and the distal $\alpha \mathrm{BV}$, and the distal $\alpha B V$ also interacts with MhuD. The proximal $\alpha B V$ forms five electrostatic and eleven hydrophobic interactions with MhuD residues. The aBV propionate- 6 carboxylate group hydrogen-bonds (H-bonds) with the backbone amides of Val83 and Ala84 (2.9 and 2.6 A, respectively), and forms a salt-bridge with the $\mathrm{NH} 1$ group of $\operatorname{Arg} 22$ (2.7 $\AA$ ). Additionally, Trp66 NE1 H-bonds (2.7 A) with the A-ring lactam oxygen (=0), and Asn7 ND2 H-bonds $(2.7 \AA)$ to the D-ring lactam oxygen (Figure $3 \mathrm{C}$ ). The tetrapyrrole plane of the distal $\alpha \mathrm{BV}$ is nearly parallel to that of the proximal $\alpha \mathrm{BV}$.

The proximal and distal $\alpha \mathrm{BV}$ molecules interact through hydrophobic and van der Waals interactions only. The distal $\mathrm{\alpha BV}$ is rotated $\sim 205^{\circ}$ and flipped $\sim 180^{\circ}$ with respect to the $\alpha \mathrm{BV}$ tetrapyrrole plane, and the distal $\alpha \mathrm{BV}$ tetrapyrrole plane is positioned $\sim 3.4 \AA$ above and translated $\sim 5.0 \AA$ relative to the proximal $\alpha B V$ plane. Consequently, the C-ring of the distal $\alpha B V$ is partially placed over the A-ring of the proximal $\alpha B V$ resulting in the distal $\alpha B V$ being more solvent exposed than the proximal one (Figure 3D). The proximal $\alpha B V$ forms five $\mathrm{H}$-bonds with MhuD whereas the distal $\alpha \mathrm{BV}$ forms only three and has fewer hydrophobic interactions with MhuD than the proximal $\alpha \mathrm{BV}$ (Figure 3D). The A-ring lactam oxygen of the distal aBV $\mathrm{H}$-bonds with Arg79 $\mathrm{NE}$ and $\mathrm{NH} 1$ (3.1 and $2.7 \AA$, respectively), and the distal $\alpha \mathrm{BV}$ propionate- $6 \mathrm{H}$-bonds to the Leu89 amide group (3.2 $\AA$ ).

Along with the proximal and distal aBV molecules, there is a third 'bridging' $\alpha \mathrm{BV}$, which connects the two symmetrical promoters each bound to two aBV molecules (Figure 3A). The third 'bridging' $\alpha B V$ is parallel to the tetrapyrrole plane of the flanking distal $\alpha B V$ molecules from each monomer and is separated by $\sim 3.8 \AA$ from each distal $\alpha B V$. 
Additionally, the bridging $\alpha \mathrm{BV}$ is rotated $\sim 90^{\circ}$ and translated $\sim 2.5 \AA$ with respect to the distal $\alpha B V$ molecules. The bridging $\alpha B V$ interacts with the distal $\alpha B V s$ by hydrophobic and van der Waals interactions, and also forms one $\mathrm{H}$-bond to each, where the bridging $\alpha B V$ ring-D/A lactam oxygen $\mathrm{H}$-bonds to the ring- $\mathrm{A} / \mathrm{D}$ pyrrole nitrogen of the distal $\alpha B V s$ $(2.8 \AA$ and $3.3 \AA$ ), respectively. Finally, the bridging aBV forms a $\mathrm{H}$-bond to each MhuD promoter, ring-A/D lactam oxygen $\mathrm{H}$-bonds with $\mathrm{Arg} 79 \mathrm{NH} 2$ group on respective monomers (3.0 and 2.6 $\AA$, respectively).

\section{Comparison of MhuD substrate and product bound structures}

There are several distinct structural differences between the inactive substrate-(MhuDheme-CN) (Graves et al., 2014) and product-bound (MhuD-R26S-aBV) MhuD structures, despite an RMSD of $1.1 \AA$ (Figure 4A). The most notable difference between the two structures is within $\alpha$-helix-2 and sequential loop region L2. In MhuD-heme-CN, the $\alpha-$ helix-2 is kinked after residue Asn68 and terminates at His75, where this region in the MhuD-R26S-aBV structure has a looser helical geometry. This kinked helical region in the MhuD-heme-CN structure positions the catalytic His75 within the active site so it coordinates with heme-iron. However, in MhuD-R26S-aBV, His75 flips out of the active site $\left(90^{\circ}\right.$ rotation and translation of $2.8 \AA$ of the $\mathrm{C} \alpha$, Figure $\left.4 \mathrm{Bi}\right)$ and is stabilized by a $\mathrm{H}$ bond between the His75 imidazole nitrogen to the backbone carbonyl of Ala27 (3.2 A). In combination with the slight unraveling of the C-terminus of $\alpha$-helix-2 in MhuD-R26S$\alpha B V$, the extended loop $L 2$ region has an additional $\alpha$-helix ( $\alpha 3$, Ala76-Asn81) as compared to MhuD-heme-CN (Graves et al., 2014) (Figure 4A). Within the L2 loop region of MhuD-heme-CN, both His78 and Arg79 are solvent-exposed with no observable electron density for the Arg79 side-chain. In contrast, in MhuD-R26S-aBV, Arg79 is located in $\alpha$-helix-3, and its side-chain is positioned towards the active site and is stabilized by a H-bond between its $\mathrm{NH1}$ group with the backbone carbonyl of Ile72 (3.1 Å) (Figure 4Bii); notably His78 is still solvent-exposed and weakly stabilized by a cation$\pi$ interaction between its imidazole side-chain and $\operatorname{Arg} 22(3.7 \AA)$.

Another minor structural difference between substrate and product bound MhuD complexes is the unraveling of the C-terminal a-helix-1 in MhuD-R26S-aBV compared to 
MhuD-heme-CN. Arg26 is situated in this location and participates in a water-mediated $\mathrm{H}$-bond with one of the heme propionates in the MhuD-heme-CN structure (Graves et al., 2014) (Figures 1B \& 4A). Within the MhuD-R26S-aBV structure, this region is no longer helical. Thus, this observed unraveling of $\alpha$-helix-1 may be the result of the Arg26Ser mutation, however, this conformational change ensures that the backbone carbonyl group of the subsequent residue, Ala27, is in $\mathrm{H}$-bonding distance of the imidazole nitrogen of His75, to stabilize the flipped out His75 in the MhuD-R26S-aBV structure (Nambu et al., 2013).

The conformational change from going from substrate to product bound also alters the active site pocket volume and the electrostatic potential of the molecular surface (Figures 4C \& 4D). The active site volume increases dramatically from heme bound to that of aBV, from 188 to $354 \AA^{3}$, calculated utilizing CASTp (Tian et al., 2018). In conjunction with the increased active site volume, the molecular surface region surrounding the active site and the L2 loop region undergoes an electrostatic potential change. The MhuD molecular surface surrounding one side of the exposed active site is positively and negatively charged in the presence of heme (Figure $4 \mathrm{C}$ ), whereas in the presence of $\alpha \mathrm{BV}$ it becomes more hydrophobic and negatively charged with a positively charged bridge capping the active site (Figure 4D). Furthermore, when rotated $90^{\circ}$ about the $y$-axis, there is an altered molecular surface electrostatics from positively charged and hydrophobic to predominately negatively charged in the presence of heme and $\alpha \mathrm{BV}$, respectively (Figures 4C \& 4D, right panels).

\section{Proximal $\alpha B V$ is representative of the MhuD physiological product}

Within the MhuD-R26S-aBV structure, the orientation of the proximal $\alpha \mathrm{BV}$ is representative of the MhuD physiological product, mycobilin. The proximal aBV adopts a similar orientation as heme in the MhuD-heme-CN structure (Figures 4A-B) (Graves et al., 2014), however $\alpha B V$ is rotated approximately $20^{\circ}$ about the plane compared to heme$\mathrm{CN}$ and the tetrapyrrole ring structure of $\alpha \mathrm{BV}$ is considerably more twisted compared to heme. Additionally, the overall conformation of MhuD proximal $\alpha B V$ is similar to the $\alpha B V$ product of $C$. diphtheriae HmuO (PDB 4GPC) (Unno et al., 2013), representative of the 
all-Z-all-syn type BV conformation. However, in another structure of hHO-1 in complex with aBV (PDB 1S8C) (Lad et al., 2004), the aBV occupies an internal cavity adjacent to the active site and exhibits a more linear extended conformation. This was proposed to be the route of $\alpha B V$ dissociation from hHO-1 in the absence of BVR (Lad et al., 2004). As $\alpha B V$ is bound tightly to MhuD, we did not anticipate that we would observe a partially dissociated product conformation or location, as seen in the $\mathrm{hHO}-1$ structure. Thus these observations suggest that the proximal $\alpha B V$ within MhuD-R26S-aBV structure is the physiological orientation of the MhuD tetrapyrrole product, mycobilin, within the active site.

\section{Simulations}

MD simulations were performed to gain further insight into the protein conformational changes associated with heme and $\alpha \mathrm{BV}$ binding. In particular, we wanted to evaluate the in silico stability of the MhuD a-helix-3 (Ala76-Asn81) in the presence of proximal aBV alone. MD simulations were set up using the biological dimer of the MhuD-R26S-aBV structure with the R26S mutation modeled as WT Arg26 and only the proximal aBVs retained. Over the $1 \mu$ s of combined simulation time, including a continuous 600 ns simulation, the $\alpha$-helix-3 persisted for over $70 \%$ of the run, suggesting that the $\alpha$-helix-3 is stable when there is just one $\alpha B V$ molecule present per active site. Interestingly, we also observe some helix formation within the $\mathrm{L} 1$ loop region, an otherwise highly flexible region of the protein (Figure 5A).

To test if the a-helix-3 forms during turnover from heme to product, two more sets of simulations were carried out. The first contained the MhuD-heme-CN structure without the cyano group (MhuD ${ }^{4 N L 5}$-heme) while the second was comprised of the MhuD ${ }^{4 N L 5}$ protein structure with $\alpha B V$ docked in place of heme to give $M_{h u D}^{4 N L 5}-\alpha B V$. As with MhuD$\alpha \mathrm{BV}$, these simulations each ran for a total of $1 \mu \mathrm{s}$. Although the two $\alpha \mathrm{BV}$ MD systems (MhuD- $\alpha \mathrm{BV}$ and $\mathrm{MhuD}^{4 \mathrm{NL5}}-\mathrm{\alpha BV}$ ) are identical in composition, they have distinct initial positions and velocities; MhuD- $\alpha B V$ simulations start from the coordinates of the $\alpha B V-$ bound crystal structure while MhuD ${ }^{4 N L 5}$ - $\alpha B V$ simulations start from those of the hemebound structure. Given sufficient simulation time, the dynamics of these two systems 
should eventually converge. While we did not reach convergence, it is compelling that we see some de novo formation of the $\alpha$-helix-3 in our simulation of MhuD ${ }^{4 N L 5}$ - $\alpha B V$ (Figure 5B), suggesting that it is a relevant structural motif that forms in the presence of $\alpha B V$. We suspect that the $\alpha$-helix-3 may be further stabilized by contact of MhuD with other interacting proteins. This $\alpha$-helix-3 is also transiently observed in the MhuD ${ }^{4 N L 5}$-heme simulation (Figure $\mathbf{5 C}$ ); however its occurrence is less frequent compared to MhuD ${ }^{4 N L 5}$ aBV.

We also sought to evaluate whether the $90^{\circ}$ rotation of the catalytic His75 side chain away from the active site, as observed in the MhuD- $\alpha B V$ structure (Figures $4 \mathbf{B i} \& \mathbf{6}$ ), is facilitated by substrate turnover to $\alpha B V$. In the MhuD- $\alpha B V$ simulations, His75 residue remains in the "flipped out" or solvent exposed orientation (Figure 6B) while in the MhuD ${ }^{4 N L 5}$-heme simulations, it is ligated to the heme-iron atom and thus remains tethered to the active site. For MhuD ${ }^{4 N L 5}-\alpha B V$, the His75 alternates between the two orientations (Figure 6C), as shown in Figures 6A \& 6B. The solvent exposed position may be further stabilized in the MhuD ${ }^{4 N L 5}-\alpha B V$ simulations after extended simulation time and upon full formation of the $\alpha-$ helix-3, as observed in the MhuD- $\alpha B V$ structure, where the Arg79 sidechain forms a hydrogen bond with the backbone carbonyl of lle72 (Figure 4Bii).

Because the Arg79 side-chain is unresolved in the MhuD ${ }^{\text {NL5}}$-heme structure but stabilized in the MhuD-aBV structure, we were also interested in exploring its dynamics in the presence of heme versus $\alpha \mathrm{BV}$. Upon inspection of the MhuD ${ }^{4 N L 5}$-heme simulations, four classifications of Arg79 positions were identified: helix 1 and helix 2 (interacting with residues on $\alpha$-helix-1 or on $\alpha$-helix-2), active site (interacting with heme or $\alpha \mathrm{BV}$ ) and solvent exposed (Figures 7A-D). In the MhuD ${ }^{4 N L 5}$-heme simulations, the varied distribution of the Arg79 positions mirrors its disorder in the crystal structure (Figure 7E). Unexpectedly, we found at times that the Arg79 forms $\mathrm{H}$-bonds with the propionate groups of the heme; in fact, in the extended 600ns simulation of MhuD ${ }^{N L 5}$-heme, the Arg79 residue for one MhuD subunit flipped into the active site and remained coordinated to the heme ligand for over $90 \%$ of the simulation (data not shown). In simulations with $\mathrm{aBV}$, Arg79 interacts with residues on helix 2 or becomes solvent exposed. Consistent 
with the MhuD- $\alpha B V$ structure, the predominant state in the MhuD- $\alpha B V$ simulations shows it interacting with helix 2 (Figure 7F). In our MhuD ${ }^{4 N L 5}-\alpha B V$ simulation, the favored state is solvent exposed (Figure 7G), but we suspect the interaction of Arg79 with helix 2 may be further stabilized upon full formation of $\alpha$-helix-3. Given that we only observe Arg79 interacting with the ligand propionate groups in simulations where His75 is oriented into the active site, we hypothesize that Arg79 plays a critical role in promoting catalysis (when heme is present) and facilitating product egress (via formation of a-helix-3).

Together these results suggest that in the MhuD-R26S- $\alpha$ BV structure we are (1) observing the proximal $\alpha B V$ in a location equivalent to the turnover product, (2) that the additional $\alpha$-helix-3 present in the MhuD-product complex is not a crystallographic artifact and (3) the $90^{\circ}$ rotation of His 75 out of the active site is consistent with substrate turnover.

\section{Discussion}

Within the PDB there is only one other protein structure with a strikingly similar $\alpha B V$ stacking conformation in its active site, a BVR from cyanobacteria (Takao et al., 2017). As with the MhuD-R26S- $\alpha$ BV structure, the BVR- $a B V$ structure has offset nearly parallel tetrapyrrole planes stacked at van der Waals distance and although the proximal and distal $\alpha B V$ molecules in MhuD have no inter-molecular $\mathrm{H}$-bonds, the BVR $\alpha \mathrm{BV}$ molecules form an intermolecular $\mathrm{H}$-bond between the lactam oxygen and pyrrole nitrogen, as observed between the distal and 'bridging' $\alpha B V$ molecules in the MhuD-R26S-aBV asymmetric unit (Figure $3 \mathbf{A}$ ).

Tetrapyrrole stacking is sometimes important for enzyme activity, however it has also been observed that heme-heme stacking can be the product of crystallization. Heme stacking has previously been shown to be involved in protein electron transfer reactions, for example, the NapB, a cytochrome subunit of nitrate reductase requires two stacked heme molecules for electron transfer (Brige et al., 2002). More recently it was demonstrated that the cyanobacterial BVR required two stacked BVs to reduce BV to bilirubin (Takao et al., 2017) by an unprecedented mechanism. In contrast, MhuD can 
also accommodate two stacked heme molecules per active site, although this renders the enzyme inactive (Chim et al., 2010). Tetrapyrrole stacking at the crystallographic interface is not unprecedented, as observed in the structures of MhuD-diheme (Chim et al., 2010), and the ChaN-heme (Chan et al., 2006), an iron-regulated lipoprotein implicated in heme acquisition in Campylobacter jejuni. As MhuD is known to be active only in its monoheme form (Chim et al., 2010), and thus only produces one molecule of product per active site, we hypothesize that the proximal $\alpha \mathrm{BV}$ is in the correct orientation of the MhuD tetrapyrrole product; consequently, the three additional stacked aBV molecules adjoining the two active sites of adjacent MhuD promoters are likely a product of crystallization.

The conformational changes between the MhuD substrate and product bound structures, while relatively subtle based on RMSD, are significant compared to those of canonical HOs. These differences are borne out in the fluctuation of the active site volume (Figure 8A). The structures of $C$. diphtheriae $\mathrm{HO}(\mathrm{HmuO})$ in its substrate- and product-bound form (PDB 1IW0, 4GPC) show some shifting and unwinding of the active site proximal and distal helices (Unno et al., 2013), while the change in the active site pocket volume is trivial (from $\sim 250 \AA^{3}$ to $\sim 260 \AA^{3}$ ) (Tian et al., 2018). By comparison, MhuD demonstrates much greater conformational versatility. When MhuD binds one heme molecule in its active form, the $\mathrm{C}$-terminal of $\alpha$-helix-1 unravels to accommodate the heme molecule and the C-terminal region of a-helix-2 extends to all the catalytically essential His75 to coordinate heme-iron, resulting in a kinked $\alpha$-helix-2. The MhuD-monoheme complex can also bind another molecule of heme resulting in its diheme inactive form, whereby the kinked $\alpha$-helix-2 is now extended and His75 binds to heme-iron of the solvent exposed heme molecule, nearly tripling the volume of the active site from $\sim 190 \AA^{3}$ to $\sim 530 \AA^{3}$ compared to the monoheme structure (Tian et al., 2018). Alternatively, when MhuDmonoheme turns over in the presence of an electron source, it forms the MhuD-product structure, which leads to the further unraveling of the C-terminal of $\alpha$-helix-1 and the kinked $\alpha$-helix-2 along with the formation of $\alpha$-helix-3. With the transformation of substrate to product, the active site of MhuD doubles in volume, from $\sim 190 \AA^{3}$ to $\sim 360 \AA^{3}$ (Tian et al., 2018). The four different conformational states of MhuD highlight this protein's inherent flexibility (Figure 8B), which may be harnessed to produce MhuD inhibitors. 
Little is known about the fate of IsdG-like protein products; but removal of their tetrapyrrole products requires protein denaturation (Nambu et al., 2013; Reniere et al., 2010). Indeed, our previous and current work suggest that both MhuD substrate and product bind in the low nanomolar range and therefore the displacement of product by substrate would only occur at high heme concentrations in vivo (Thakuri et al., 2018). We propose three possible mechanisms of MhuD product release; (1) a dramatic conformational change would reduce product affinity and result in dissociation, (2) IsdG-type proteins are 'suicide' proteins that after one turnover require degradation or (3) an accessory protein is required for the removal of product from the MhuD active site. Although MhuD is an inherently flexible protein, as described above, its high affinity for tetrapyrroles decreases the likelihood that a conformational change alone would promote product release. Because it has been shown that $S$. aureus IsdG is degraded in vivo in its apo form, yet stabilized in the presence of heme (Reniere et al., 2011), it seems unlikely that IsdG-type enzymes are also degraded when bound to product. As the MhuD-R26S-aBV complex structure has a newly formed structural element and an associated shift in molecular surface electrostatics in comparison to MhuD-monoheme complex, these conformational changes may promote protein-protein interactions to aid in product removal.

Protein-protein interaction-induced product removal is reminiscent of human HO-1 BV removal. Human BVR interacts with $\mathrm{hHO}-1$, albeit via a weak interaction, to remove the product BV and further reduce it to bilirubin (Maines and Trakshel, 1993). In contrast, the well-studied bacterial $\mathrm{HO}$ from $P$. aeruginosa excretes BV without further reduction (Barker et al., 2012). We hypothesize that a yet-to-be-identified Mtb protein removes product from MhuD and potentially aids in its eventual excretion, as observed in the human $\mathrm{HO}$ system. Surprisingly, Mtb has four close homologs of BVR even though Mtb does not have a conventional BV-producing $\mathrm{HO}$ enzyme (Chim et al., 2010). One of these homologs, Rv2074, has BV reduction activity although its electron donating cofactor is the flavin cofactor F420 (Ahmed et al., 2016), a deazaflavin cofactor that is a low potential hydride transfer agent (Bashiri et al., 2019), rather than flavin mononucleotide (FMN) as observed for eukaryotic BVRs (Sugishima et al., 2018). In contrast, Rv1155 
does not readily reduce BV (Ahmed et al., 2015). Consequently, it was proposed that one of the BV inactive Mtb BVRs catabolizes mycobilins. Rv2607 and Rv2991 have not been tested for BVR activity and could also act in MhuD product breakdown (Ahmed et al., 2015). Mtb has both heme and siderophore-mediated iron acquisition systems (Chao et al., 2019), however Mycobacterium leprea only has a heme uptake and catabolism pathway. The M. leprea proteome only has the Mtb BVR homologs, Rv1155 and Rv2607, suggesting one of these proteins is perhaps involved in MhuD product removal.

Finally, the MhuD-product complex has an additional $\alpha$-helix-3 and an accompanying change in electrostatic surface potential, compared to the MhuD-substrate complex, which may be essential for mediating protein-protein interactions to promote product

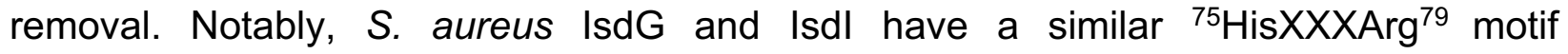
encompassing the a-helix-3 (Figure 9), suggesting that both $S$. aureus IsdG and Isdl also form the new secondary structure element upon product formation; this may be a common structural feature among all IsdG-type proteins in complex with product.

\section{Acknowledgements}

We would like to thank Tom Poulos for discussions and critical reading of the manuscript, as well as Xiaorui Chen for advice on the more difficult aspects of structure refinement. We thank the Advanced Light Source at Berkeley National Laboratories (ALS) and the Stanford Synchrotron Radiation Lightsource (SSRL) for their invaluable help in data collection.

\section{Funding}

C.W.G. thanks the National Institutes of Health (NIH) for financial support (P01Al095208), A.C. thanks the National Science Foundation for predoctoral fellowship support (DGE-1321846), and K.H.B. thanks the $\mathrm{NIH}$ for support from a predoctoral training grant (T32GM108561). 


\section{Notes}

The authors declare no competing financial interests.

\section{Accession IDs}

hHO-1 P09601

HmuO Q54Al1

MhuD P9WKH3

IsdG Q7A649

Isdl Q7A827

\section{References}

Adams, P.D., Afonine, P.V., Bunkoczi, G., Chen, V.B., Davis, I.W., Echols, N., Headd, J.J., Hung, L.W., Kapral, G.J., Grosse-Kunstleve, R.W., et al. (2010). PHENIX: a comprehensive Python-based system for macromolecular structure solution. Acta Crystallogr D Biol Crystallogr 66, 213-221.

Ahmed, F.H., Carr, P.D., Lee, B.M., Afriat-Jurnou, L., Mohamed, A.E., Hong, N.S., Flanagan, J., Taylor, M.C., Greening, C., and Jackson, C.J. (2015). SequenceStructure-Function Classification of a Catalytically Diverse Oxidoreductase Superfamily in Mycobacteria. J Mol Biol 427, 3554-3571.

Ahmed, F.H., Mohamed, A.E., Carr, P.D., Lee, B.M., Condic-Jurkic, K., O'Mara, M.L., and Jackson, C.J. (2016). Rv2074 is a novel F420 H2 -dependent biliverdin reductase in Mycobacterium tuberculosis. Protein Sci 25, 1692-1709.

Barker, K.D., Barkovits, K., and Wilks, A. (2012). Metabolic flux of extracellular heme uptake in Pseudomonas aeruginosa is driven by the iron-regulated heme oxygenase (HemO). J Biol Chem 287, 18342-18350.

Bashiri, G., Antoney, J., Jirgis, E.N.M., Shah, M.V., Ney, B., Copp, J., Stuteley, S.M., Sreebhavan, S., Palmer, B., Middleditch, M., et al. (2019). A revised biosynthetic pathway for the cofactor F420 in prokaryotes. Nat Commun 10, 1558.

Battye, T.G., Kontogiannis, L., Johnson, O., Powell, H.R., and Leslie, A.G. (2011). iMOSFLM: a new graphical interface for diffraction-image processing with MOSFLM. Acta Crystallogr D Biol Crystallogr 67, 271-281. 
Brige, A., Leys, D., Meyer, T.E., Cusanovich, M.A., and Van Beeumen, J.J. (2002). The 1.25 A resolution structure of the diheme NapB subunit of soluble nitrate reductase reveals a novel cytochrome c fold with a stacked heme arrangement. Biochemistry 41 , 4827-4836.

Brouard, S., Otterbein, L.E., Anrather, J., Tobiasch, E., Bach, F.H., Choi, A.M., and Soares, M.P. (2000). Carbon monoxide generated by heme oxygenase 1 suppresses endothelial cell apoptosis. J Exp Med 192, 1015-1026.

Case, D.A., Cerutti, D.S., Cheatham III, T.E., Darden, T.A., Duke, R.E., Giese, T.J., Gohlke, H., Goetz, A.W., Greene, D., Homeyer, N., et al. (2015). AmberTools15.

Chan, A.C., Lelj-Garolla, B., F, I.R., Pedersen, K.A., Mauk, A.G., and Murphy, M.E. (2006). Cofacial heme binding is linked to dimerization by a bacterial heme transport protein. J Mol Biol 362, 1108-1119.

Chao, A., and Goulding, C.W. (2019). A Single Mutation in the Mycobacterium tuberculosis Heme-Degrading Protein, MhuD, Results in Different Products. Biochemistry 58, 489-492.

Chao, A., Sieminski, P.J., Owens, C.P., and Goulding, C.W. (2019). Iron Acquisition in Mycobacterium tuberculosis. Chem Rev 119, 1193-1220.

Chim, N., Iniguez, A., Nguyen, T.Q., and Goulding, C.W. (2010). Unusual diheme conformation of the heme-degrading protein from Mycobacteriuim tuberculosis. Journal of Molecular Biology 395, 595-608.

Conger, M.A., Pokhrel, D., and Liptak, M.D. (2017). Tight binding of heme to Staphylococcus aureus IsdG and Isdl precludes design of a competitive inhibitor. Metallomics 9, 556-563.

Dore, S., Takahashi, M., Ferris, C.D., Zakhary, R., Hester, L.D., Guastella, D., and Snyder, S.H. (1999). Bilirubin, formed by activation of heme oxygenase-2, protects neurons against oxidative stress injury. Proc Natl Acad Sci U S A 96, 2445-2450.

Eastman, P., Swails, J., Chodera, J.D., McGibbon, R.T., Zhao, Y., Beauchamp, K.A., Wang, L.P., Simmonett, A.C., Harrigan, M.P., Stern, C.D., et al. (2017). OpenMM 7: Rapid development of high performance algorithms for molecular dynamics. PLoS Comput Biol 13, e1005659.

Ferris, C.D., Jaffrey, S.R., Sawa, A., Takahashi, M., Brady, S.D., Barrow, R.K., Tysoe, S.A., Wolosker, H., Baranano, D.E., Dore, S., et al. (1999). Haem oxygenase-1 prevents cell death by regulating cellular iron. Nat Cell Biol 1,152-157. 
Graves, A.B., Morse, R.P., Chao, A., Iniguez, A., Goulding, C.W., and Liptak, M.D. (2014). Crystallographic and spectroscopic insights into heme degradation by Mycobacterium tuberculosis MhuD. Inorg Chem 53, 5931-5940.

Harris, D., Loew, G., and Waskell, L. (2001). Calculation of the electronic structure and spectra of model cytochrome P450 compound I. J Inorg Biochem 83, 309-318.

Lad, L., Friedman, J., Li, H., Bhaskar, B., Ortiz de Montellano, P.R., and Poulos, T.L. (2004). Crystal structure of human heme oxygenase-1 in a complex with biliverdin. Biochemistry 43, 3793-3801.

Lee, W.C., Reniere, M.L., Skaar, E.P., and Murphy, M.E. (2008). Ruffling of metalloporphyrins bound to IsdG and Isdl, two heme-degrading enzymes in Staphylococcus aureus. J Biol Chem 283, 30957-30963.

Lindorff-Larsen, K., Piana, S., Palmo, K., Maragakis, P., Klepeis, J.L., Dror, R.O., and Shaw, D.E. (2010). Improved side-chain torsion potentials for the Amber ff99SB protein force field. Proteins 78, 1950-1958.

Lojek, L.J., Farrand, A.J., Wisecaver, J.H., Blaby-Haas, C.E., Michel, B.W., Merchant, S.S., Rokas, A., and Skaar, E.P. (2017). Chlamydomonas reinhardtii LFO1 Is an IsdG Family Heme Oxygenase. mSphere 2.

Lowry, O.H., Rosebrough, N.J., Farr, A.L., and Randall, R.J. (1951). Protein measurement with the Folin phenol reagent. J Biol Chem 193, 265-275.

Maines, M.D., and Trakshel, G.M. (1993). Purification and characterization of human biliverdin reductase. Arch Biochem Biophys 300, 320-326.

Mantle, T.J. (2002). Haem degradation in animals and plants. Biochem Soc Trans 30, 630-633.

Matsui, T., Nambu, S., Goulding, C.W., Takahashi, S., Fujii, H., and Ikeda-Saito, M. (2016). Unique coupling of mono- and dioxygenase chemistries in a single active site promotes heme degradation. Proc Natl Acad Sci U S A.

Matsui, T., Nambu, S., Ono, Y., Goulding, C.W., Tsumoto, K., and Ikeda-Saito, M. (2013). Heme degradation by Staphylococcus aureus IsdG and Isdl liberates formaldehyde rather than carbon monoxide. Biochemistry 52, 3025-3027.

Matsui, T., Unno, M., and Ikeda-Saito, M. (2010). Heme oxygenase reveals its strategy for catalyzing three successive oxygenation reactions. Acc Chem Res 43, 240-247.

McGibbon, R.T., Beauchamp, K.A., Harrigan, M.P., Klein, C., Swails, J.M., Hernandez, C.X., Schwantes, C.R., Wang, L.P., Lane, T.J., and Pande, V.S. (2015). MDTraj: A 
Modern Open Library for the Analysis of Molecular Dynamics Trajectories. Biophys J $109,1528-1532$.

Nambu, S., Matsui, T., Goulding, C.W., Takahashi, S., and Ikeda-Saito, M. (2013). A new way to degrade heme: the Mycobacterium tuberculosis enzyme MhuD catalyzes heme degradation without generating CO. J Biol Chem 288, 10101-10109.

Noguchi, M., Yoshida, T., and Kikuchi, G. (1979). Specific requirement of NADPHcytochrome $\mathrm{c}$ reductase for the microsomal heme oxygenase reaction yielding biliverdin IX alpha. FEBS Lett 98, 281-284.

Ratliff, M., Zhu, W., Deshmukh, R., Wilks, A., and Stojiljkovic, I. (2001). Homologues of neisserial heme oxygenase in gram-negative bacteria: degradation of heme by the product of the pigA gene of Pseudomonas aeruginosa. J Bacteriol 183, 6394-6403.

Reniere, M.L., Haley, K.P., and Skaar, E.P. (2011). The flexible loop of Staphylococcus aureus IsdG is required for its degradation in the absence of heme. Biochemistry 50 , 6730-6737.

Reniere, M.L., Ukpabi, G.N., Harry, S.R., Stec, D.F., Krull, R., Wright, D.W., Bachmann, B.O., Murphy, M.E., and Skaar, E.P. (2010). The IsdG-family of haem oxygenases degrades haem to a novel chromophore. Mol Microbiol 75, 1529-1538.

Schmitt, M.P. (1997). Utilization of host iron sources by Corynebacterium diphtheriae: identification of a gene whose product is homologous to eukaryotic heme oxygenases and is required for acquisition of iron from heme and hemoglobin. J Bacteriol 179, 838845.

Schuller, D.J., Wilks, a., Ortiz de Montellano, P.R., and Poulos, T.L. (1999). Crystal structure of human heme oxygenase-1. Nature structural biology 6, 860-867.

Skaar, E.P., Gaspar, A.H., and Schneewind, O. (2004). IsdG and Isdl, heme-degrading enzymes in the cytoplasm of Staphylococcus aureus. J Biol Chem 279, 436-443.

Sugishima, M., Wada, K., and Fukuyama, K. (2018). Recent Advances in the Understanding of the Reaction Chemistries of the Heme Catabolizing Enzymes $\mathrm{HO}$ and BVR Based on High Resolution Protein Structures. Curr Med Chem.

Takao, H., Hirabayashi, K., Nishigaya, Y., Kouriki, H., Nakaniwa, T., Hagiwara, Y., Harada, J., Sato, H., Yamazaki, T., Sakakibara, Y., et al. (2017). A substrate-bound structure of cyanobacterial biliverdin reductase identifies stacked substrates as critical for activity. Nat Commun 8, 14397.

Tenhunen, R., Marver, H.S., and Schmid, R. (1969). Microsomal heme oxygenase. Characterization of the enzyme. J Biol Chem 244, 6388-6394. 
Thakuri, B., Graves, A.B., Chao, A., Johansen, S.L., Goulding, C.W., and Liptak, M.D. (2018). The affinity of MhuD for heme is consistent with a heme degrading function in vivo. Metallomics.

Tian, W., Chen, C., Lei, X., Zhao, J., and Liang, J. (2018). CASTp 3.0: computed atlas of surface topography of proteins. Nucleic Acids Res 46, W363-W367.

Unno, M., Ardevol, A., Rovira, C., and Ikeda-Saito, M. (2013). Structures of the substrate-free and product-bound forms of $\mathrm{HmuO}$, a heme oxygenase from corynebacterium diphtheriae: x-ray crystallography and molecular dynamics investigation. J Biol Chem 288, 34443-34458.

Vanella, L., Barbagallo, I., Tibullo, D., Forte, S., Zappala, A., and Li Volti, G. (2016). The non-canonical functions of the heme oxygenases. Oncotarget 7, 69075-69086.

Wilks, A. (2002). Heme oxygenase: evolution, structure, and mechanism. Antioxid Redox Signal 4, 603-614.

Wilks, A., and Schmitt, M.P. (1998). Expression and characterization of a heme oxygenase (Hmu O) from Corynebacterium diphtheriae. Iron acquisition requires oxidative cleavage of the heme macrocycle. J Biol Chem 273, 837-841.

Wu, R., Skaar, E.P., Zhang, R., Joachimiak, G., Gornicki, P., Schneewind, O., and Joachimiak, A. (2005). Staphylococcus aureus IsdG and Isdl, heme-degrading enzymes with structural similarity to monooxygenases. J Biol Chem 280, 2840-2846.

Yoshida, T., Noguchi, M., and Kikuchi, G. (1980). Oxygenated form of heme - heme oxygenase complex and requirement for second electron to initiate heme degradation from the oxygenated complex. J Biol Chem 255, 4418-4420.

Zhu, W., Wilks, A., and Stojiljkovic, I. (2000). Degradation of heme in gram-negative bacteria: the product of the hemO gene of Neisseriae is a heme oxygenase. J Bacteriol 182, 6783-6790. 
Table 1 Heme and aBV binding affinities $\left(K_{d}\right)$ were determined for WT MhuD and the R26S variant complexes with 1:1 heme or aBV to protein ratio. Each experiment was performed in triplicate.

\begin{tabular}{|l|l|l|}
\hline & heme $K_{d}(\mathrm{nM})$ & aBV $K_{d}(\mathrm{nM})$ \\
\hline MhuD-WT & $6.0 \pm 2.9$ & $36.4 \pm 2.1$ \\
\hline MhuD-R26S & $9.5 \pm 3.0$ & $92.4 \pm 6.8$ \\
\hline
\end{tabular}


Table 2 X-ray diffraction data and atomic refinement for MhuD-R26S- $\alpha$ BV complex.

\begin{tabular}{|c|c|}
\hline Space Group & 1222 \\
\hline Unit cell dimensions $(\AA)$ & $37.22 \times 113.61 \times 113.65$ \\
\hline $\mathrm{pH}$ of crystallization condition & 6.5 \\
\hline Protein concentration $(\mathrm{mg} / \mathrm{mL})$ & 20 \\
\hline \multicolumn{2}{|l|}{ Data Collection } \\
\hline Wavelength, $\AA$ & 1.0 \\
\hline Resolution range & $35.94-2.5$ \\
\hline Unique reflections (total) & $8713(17426)$ \\
\hline Completeness, \%* & $99.89(100.00)$ \\
\hline Redundancy* & $2.0(2.0)$ \\
\hline $\mathrm{R}_{\text {merge }}{ }^{*} \dagger$ & $0.013(0.062)$ \\
\hline $\mathrm{CC}_{1 / 2}{ }^{*}$ & $1.0(0.99)$ \\
\hline $1 / \sigma^{*}$ & $25.41(10.64)$ \\
\hline NCS copies & 2 \\
\hline \multicolumn{2}{|l|}{ Model refinement } \\
\hline Resolution range, $\AA$ & $35.93-2.5$ \\
\hline No. of reflections (working/free) & $8707(867)$ \\
\hline No. of protein + ligand atoms & 1430 \\
\hline No. of water molecules & 16 \\
\hline No. of $a B V s$ in NCS & 5 \\
\hline $\mathrm{R}_{\text {work }} / \mathrm{R}_{\text {free }}, \% \pi$ & $23.16 / 28.53$ \\
\hline \multicolumn{2}{|l|}{ Rms deviations } \\
\hline Bond lengths, $\AA$ & 0.009 \\
\hline Bond angles & 1.52 \\
\hline \multicolumn{2}{|l|}{ Ramachandran plot } \\
\hline Most favorable region, $\%$ & 90.96 \\
\hline Additional allowed region, \% & 7.98 \\
\hline Outliers (Val30), \% & 1.06 \\
\hline PDB ID code & 6PLE \\
\hline
\end{tabular}

${ }^{*}$ Statistics for the highest-resolution shell are given in parentheses.

${ }^{\dagger} R_{\text {merge }}=\Sigma_{h k \mid} \Sigma_{i}\left|l_{i}(h k l)-(I(h k l))\right| / \sum_{h k \mid} \Sigma_{i} l_{i}(h k l)$

" $R_{\text {work }}=\Sigma \mid F_{\text {obs }}-F_{\text {calc }} / / \Sigma F_{\text {obs. }}$. R Rree was computed identically except where all reflections belong to a test set of $10 \%$ randomly selected data. 


\section{Figure Legends}

Figure 1 Structures of tetrapyrroles and WT-MhuD-monoheme. A. The structure of heme and heme tetrapyrrole degradation products. B. The structure of the active site of the Mtb heme-degrading protein, MhuD, in its cyano-derivatized monoheme form (cartoon, white). Depicted in stick representation (cyan) are essential Asn7, Trp66 and His75 (which coordinates heme-iron) residues, and Arg26 that forms a water-mediated $\mathrm{H}$-bond with one of the heme propionates.

Figure 2 Heme and $\alpha B V$ affinity of MhuD and the R26S variant. Representative fluorescent emission intensities at $340 \mathrm{~nm}$ after excitation at $280 \mathrm{~nm}$ of WT MhuD (left panels) and the MhuD-R26S variant (right panels) with increasing concentrations of $\mathbf{A}$. heme and B. $\alpha B V$.

Figure 3 Structure of the MhuD-R26S- $\alpha$ BV complex. A. The asymmetric unit that contains five molecules of $\alpha \mathrm{BV}$ stacked connecting the active sites of two MhuD monomers. B. The dimeric biological assembly with two molecules of $\alpha B V$ per active site. The new structural helix, $\alpha 3$, is denoted with a red arrow. C. Interactions of the proximal $\alpha \mathrm{BV}$ (orange) with MhuD (green). Blue dashed lines represent $\mathrm{H}$-bonds with their length in $\AA$. D. Interactions of the distal $\alpha B V$ (yellow) with MhuD (green).

\section{Figure 4 Structural comparison of MhuD-heme-CN and MhuD-R26S- $\alpha B V$. A.} Superposition of the MhuD-heme-CN (pink, PDB ID 4NL5, the cyano group is omitted for clarity) and MhuD-R26S- $\alpha$ BV (green). B. Active site comparison with (i) catalytic residues Asn7 and His75, and (ii) residues Asn7 and Arg79, are shown in stick representation. Black dashed lines represent H-bonds with their length in Å. C-D Electrostatic molecular surface representations, where blue and red are positively and negatively charged, respectively. Right panel is the left panel rotated $90^{\circ}$ anticlockwise (ACW). C. MhuDheme-CN (WT) and D. MhuD-R26S- $\alpha$ BV ( $\alpha B V)$. 
Figure 5 Helical stability in MD simulations of MhuD. The frequency of helix formation (DSSP) is plotted for each residue of MhuD in simulations with $\alpha \mathrm{BV}$ and heme. For simulations of MhuD- $\alpha B V$ initiated from the coordinates of the MhuD- $\alpha B V$ structure, the novel $\alpha$-helix ( $\alpha 3$ ) that forms in $L 2$ persists. In simulations of MhuD ${ }^{4 N L 5}-\alpha B V$ initiated from the coordinates of the MhuD-monoheme structure (PDB: 4NL5), $\alpha 3$ transiently forms in the $\mathrm{L} 2$ region. In the presence of heme (MhuD ${ }^{4 N L 5}$-heme), formation of $\alpha 3$ also is observed but less frequently than in MhuD ${ }^{4 N L 5}-\alpha B V$.

Figure 6 Orientation of His75 in MD simulations of MhuD. During MD simulations of MhuD with $\alpha \mathrm{BV}$, the position of His75 can be classified as either a) directed into the active site or b) rotated out of the active site. This designation was assigned based on the distance between the His75 $\varepsilon$ nitrogen atom and one of the $\alpha \mathrm{BV}$ nitrogens, as described in the methods section. The structure in $A$. is a snapshot from the $M h u D^{4 N L 5}-\alpha B V$ simulation while $B$. shows a snapshot of the MhuD- $\alpha B V$ simulation. The frequency of each position is plotted for the MhuD- $\alpha$ BV simulations initiated from C. the MhuD-R26S$\alpha B V$ crystal structure and $D$. the $M{ }^{4 N L 5}-\alpha B V$ simulations, where $\alpha B V$ has docked in place of heme in the MhuD-monoheme structure. For the MhuD- $\alpha$ BV simulations, His75 remains oriented out of the active site while in the $M D$ of $M h u D^{4 N L 5}-\alpha B V$, His75 flips in and out of the active site. Notably, data for MhuD ${ }^{4 N L 5}$-heme simulations of the MhuDmonoheme structure is not shown as the His75 residue is ligated to the heme-iron and thus cannot explore alternate positions.

Figure 7 Position of Arg79 side-chain during MD simulations of MhuD. In the MhuDmonoheme structure (PDB ID 4NL5), the Arg79 side-chain is unresolved. MD simulations of the MhuD- monoheme structure (MhuD ${ }^{4 N L 5}$-heme) highlight the flexibility of this residue. We have classified its positions during simulations into four categories: A. Helix 1 shows Arg79 interacting with $\alpha$-helix-1 (residues 16-25), B. Solvent Exposed refers to Arg79 freely oriented in the surrounding water, C. Active Site is where Arg79 interacts with the ligand (heme or $\alpha \mathrm{BV}$ ), and D. Helix 2 refers to the Arg79 side-chain interacting with residues in $\alpha$-helix-2 (residues 60-75). All representations in A-D are snapshots from 
the simulation of MhuD ${ }^{4 N L 5}$-heme. The frequency of each Arg79 position is plotted in panels E-G for all three simulation types. For E. MhuD ${ }^{4 N L 5}$-heme, Arg79 is highly motile and at times, coordinates with the propionate groups of the heme ligand. For F. MhuD$\alpha B V$, where simulations are initiated from the coordinates of the MhuD- $\alpha B V$ structure, Arg79 predominantly interacts with helix 2 residues and may be stabilized by its participation in $\alpha$-helix-3. For $\mathbf{G}$. MhuD ${ }^{4 N L 5}-\alpha B V$, where heme has been removed from the MhuD-monoheme structure and $\alpha \mathrm{BV}$ is docked in its place, the Arg79 remains mostly solvent exposed.

Figure 8 Depiction of MhuD's conformational flexibility along its reaction pathway. A. Superposition of apo-MhuD (white, PDB ID: 5UQ4), MhuD-monoheme (cyan, PDB ID: 4NL5), MhuD-diheme (pink, PDB ID: 3HX9) and MhuD-R26S- $\alpha$ BV (blue). B. Apo-MhuD binds heme to form the closed MhuD-monoheme active form and in the presence of an electron donor it can degrade heme to the product bound form or alternatively, if there are high concentrations of heme, then it forms an open diheme inactive form.

Figure 9 Structure- and sequence-based alignment of MhuD and S. aureus IsdG and Isdl. Structure-based alignment of MhuD, IsdG and Isdl. The secondary structural elements (arrows are $\beta$-strands and waves are $\alpha$-helices) are based on the MhuDmonoheme structure (PDB ID: 4NL5), apart from the purple cylinder $\alpha$-helix-3, that is the new helix observed in the MhuD-R26S- $\alpha$ BV structure. Important conserved residues are

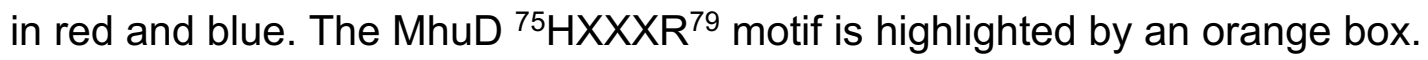




\section{Figure 1}

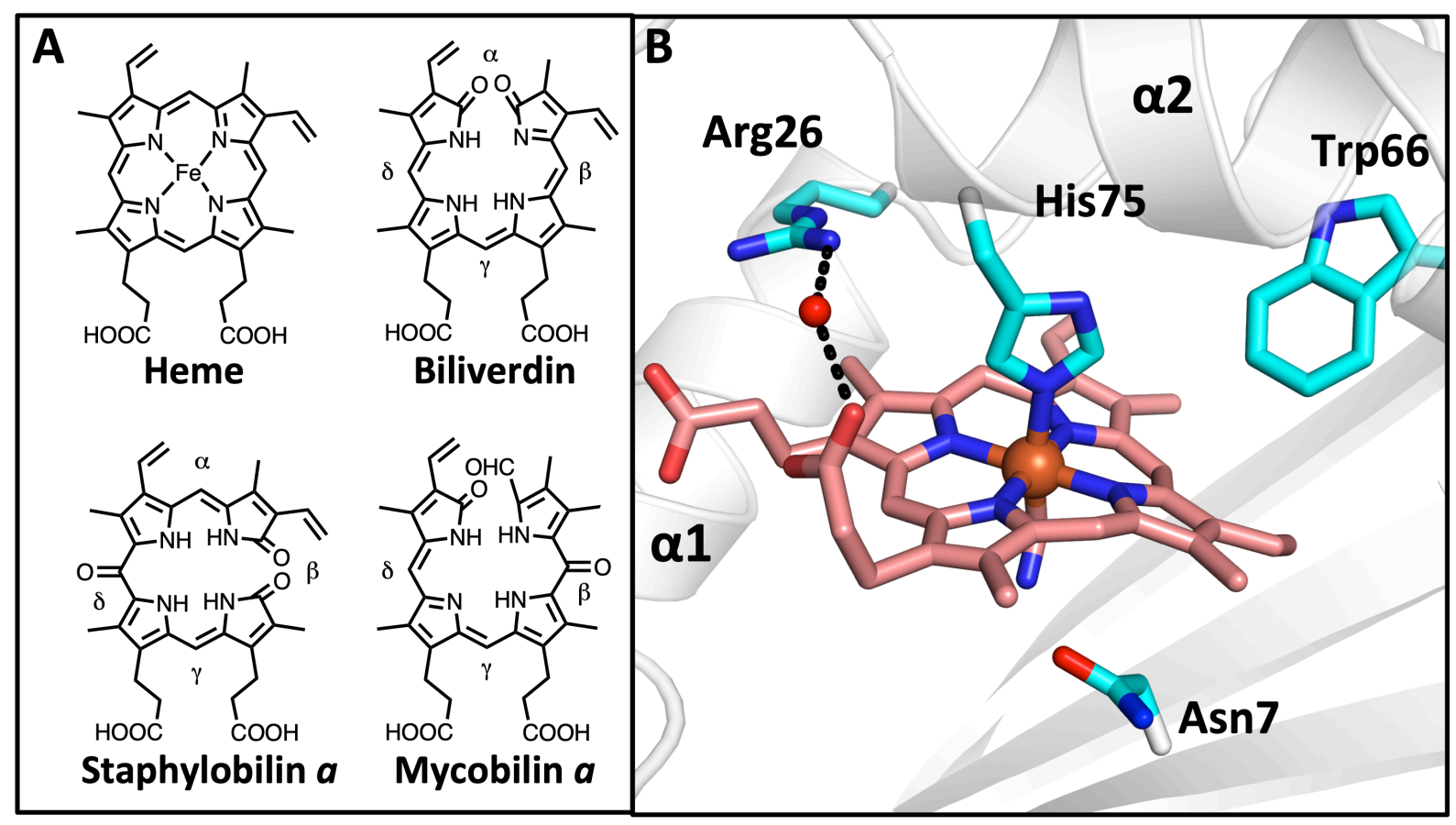




\section{Figure 2}
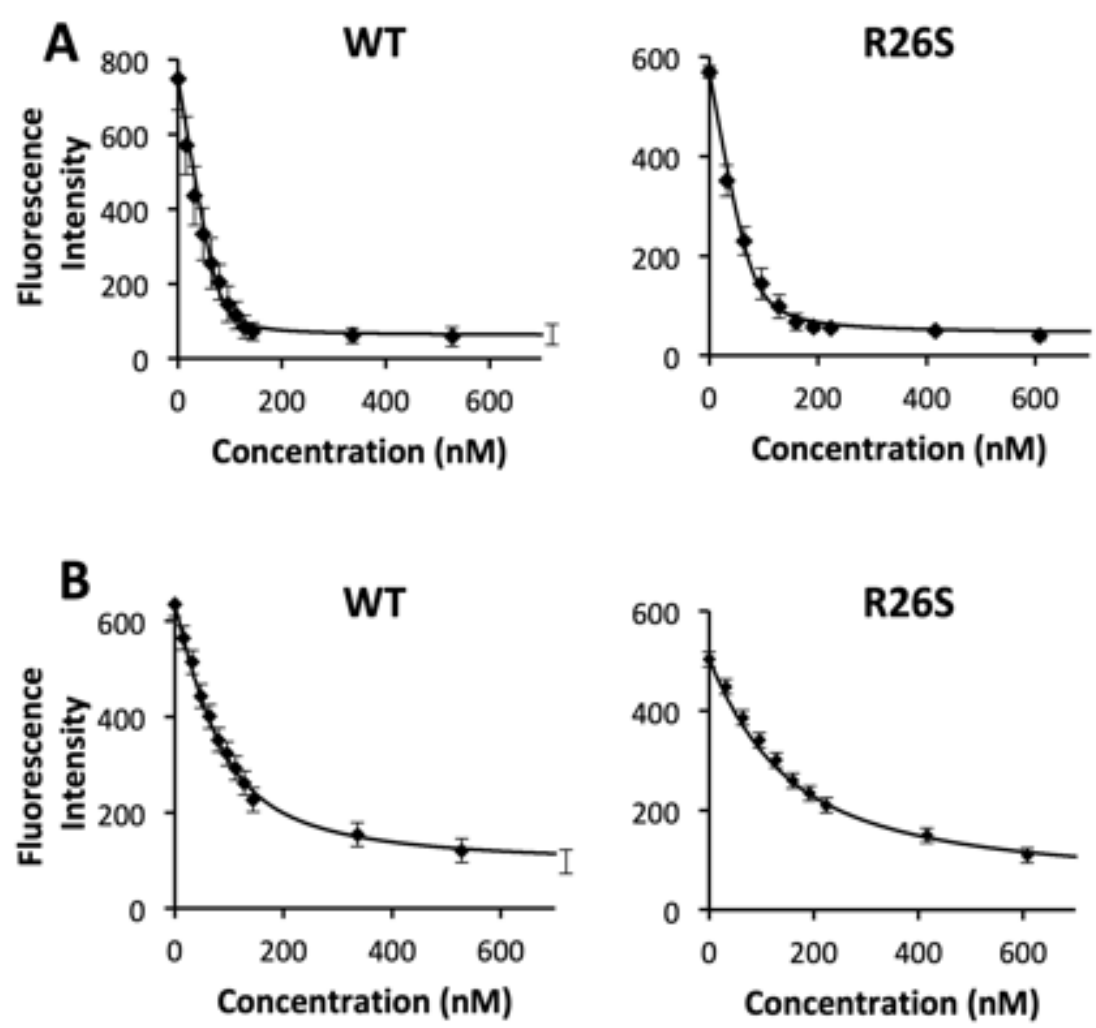


\section{Figure 3}
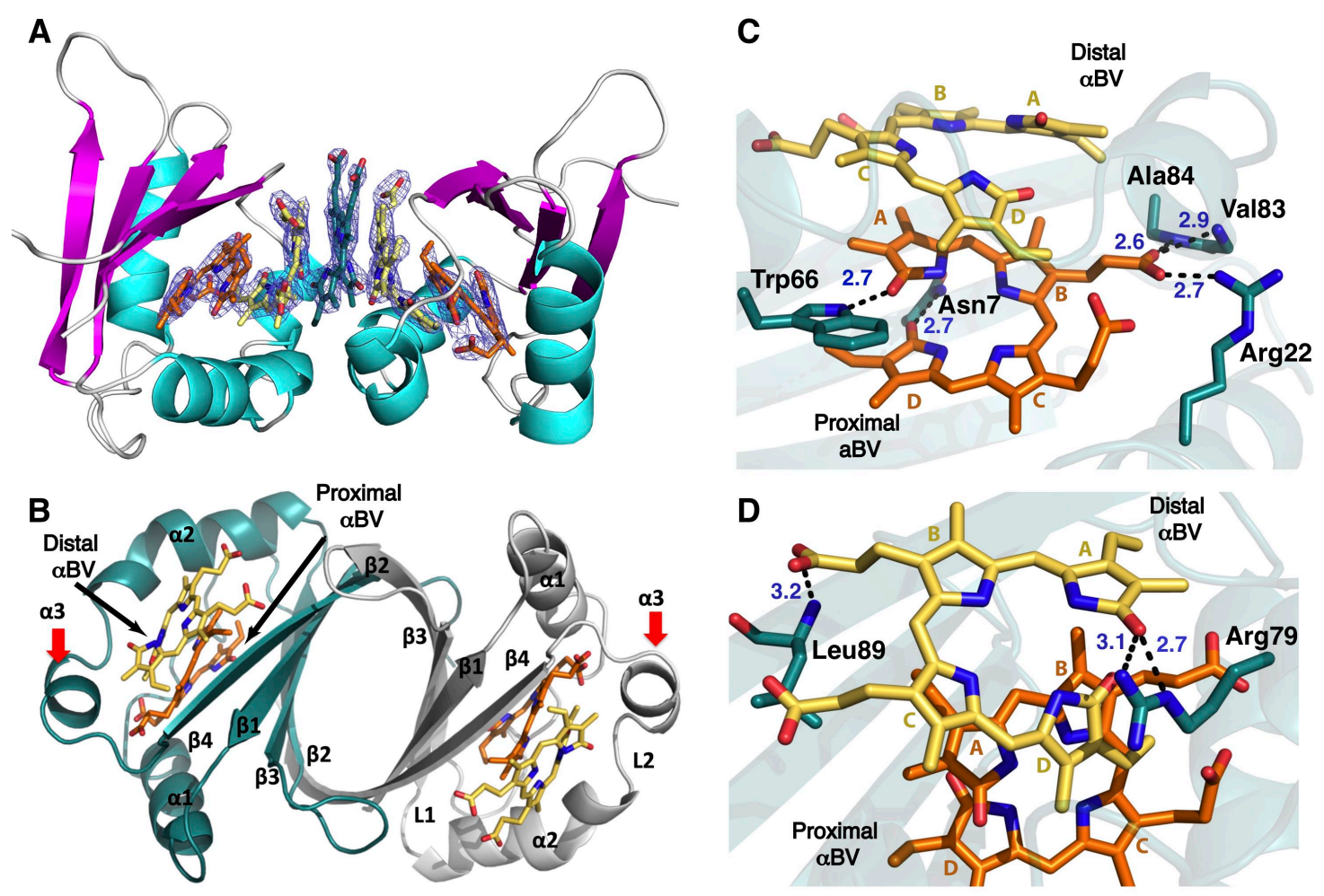


\section{Figure 4}

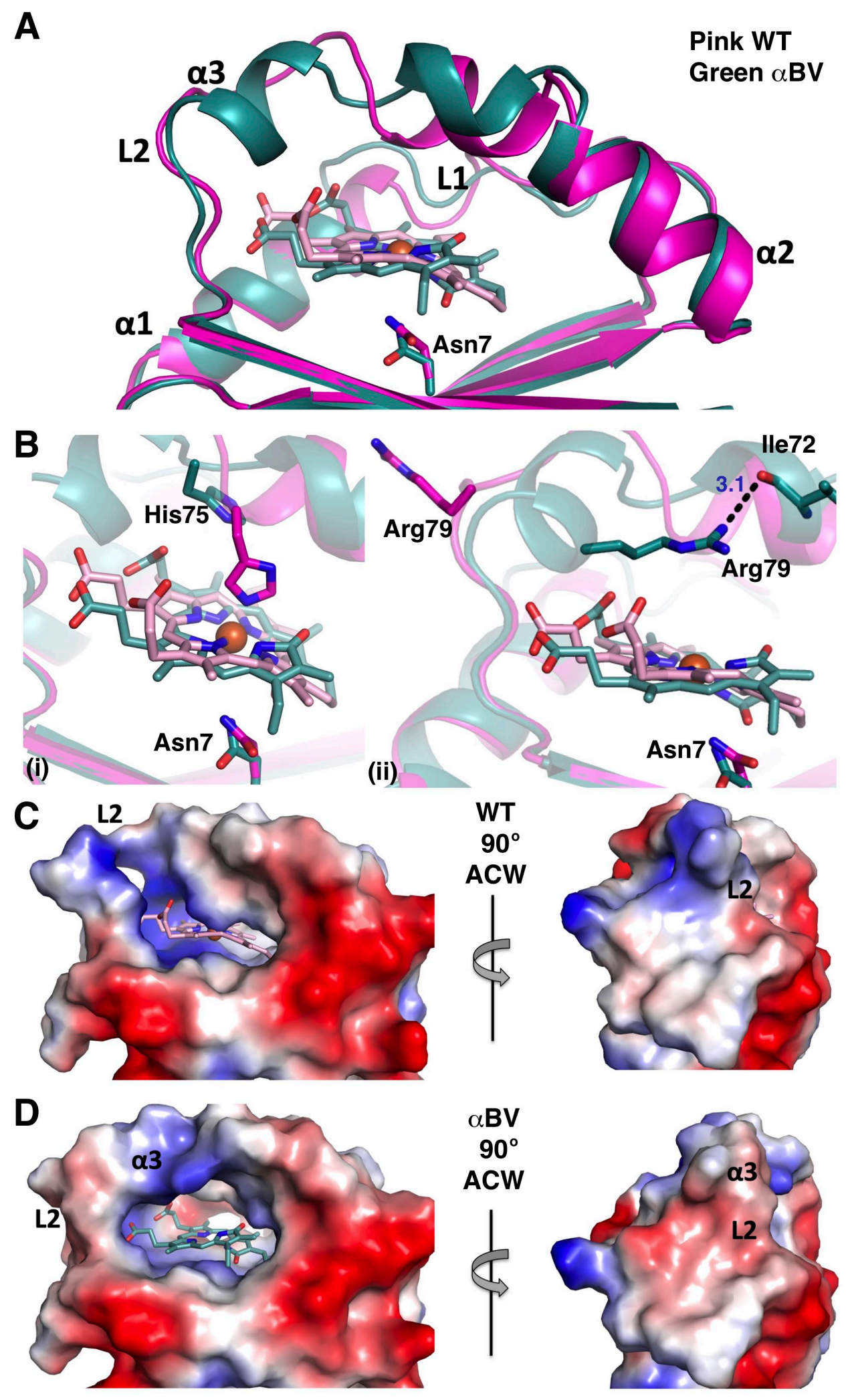




\section{Figure 5}

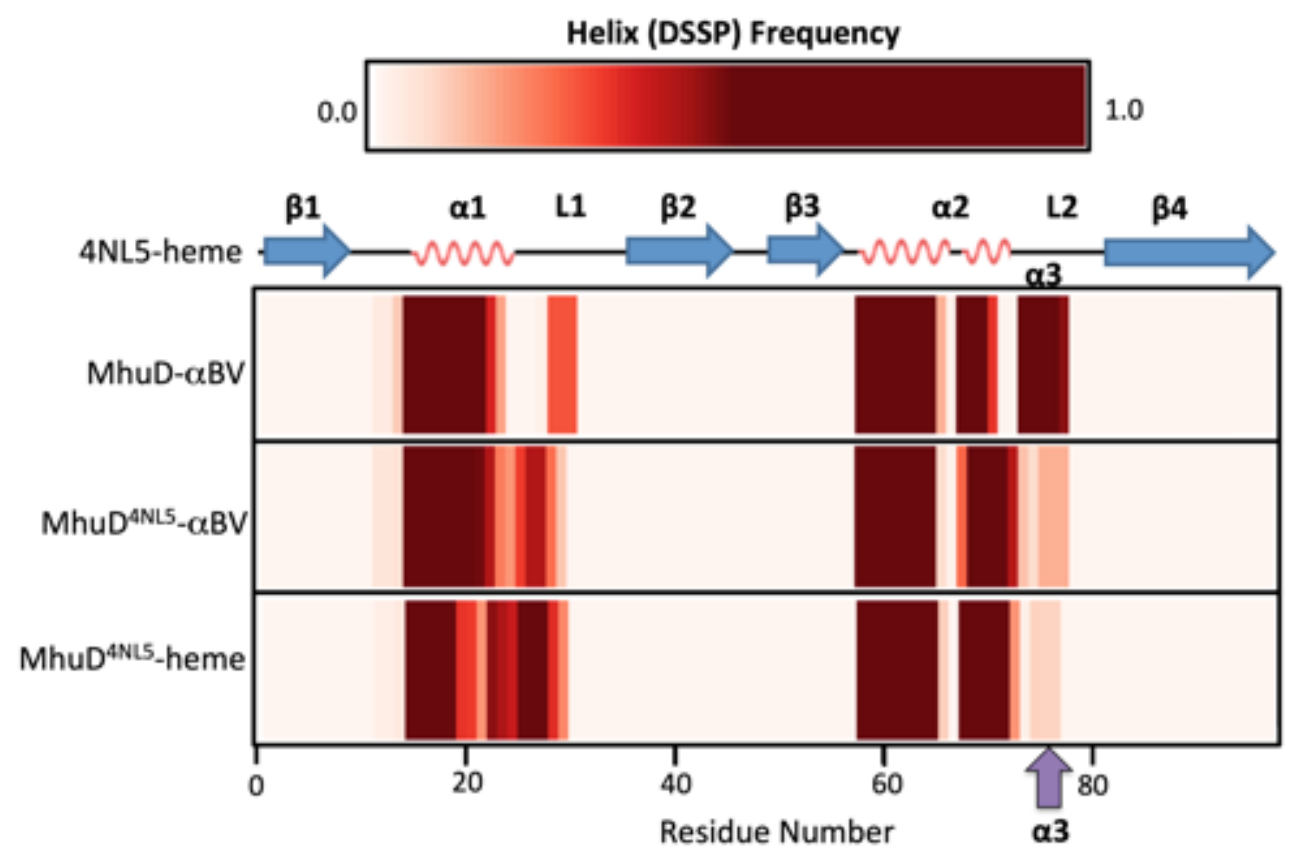


Figure 6

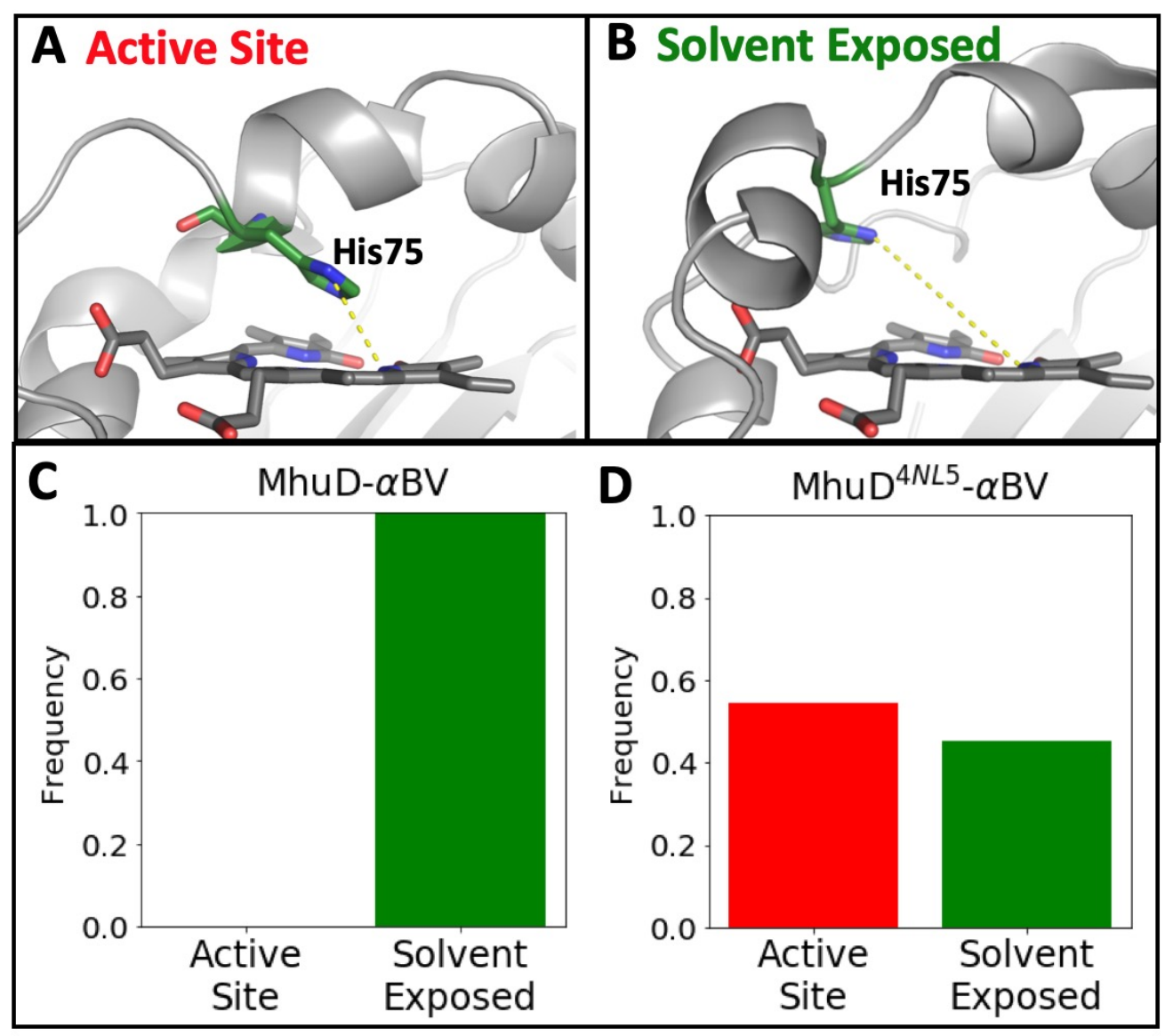


Figure 7

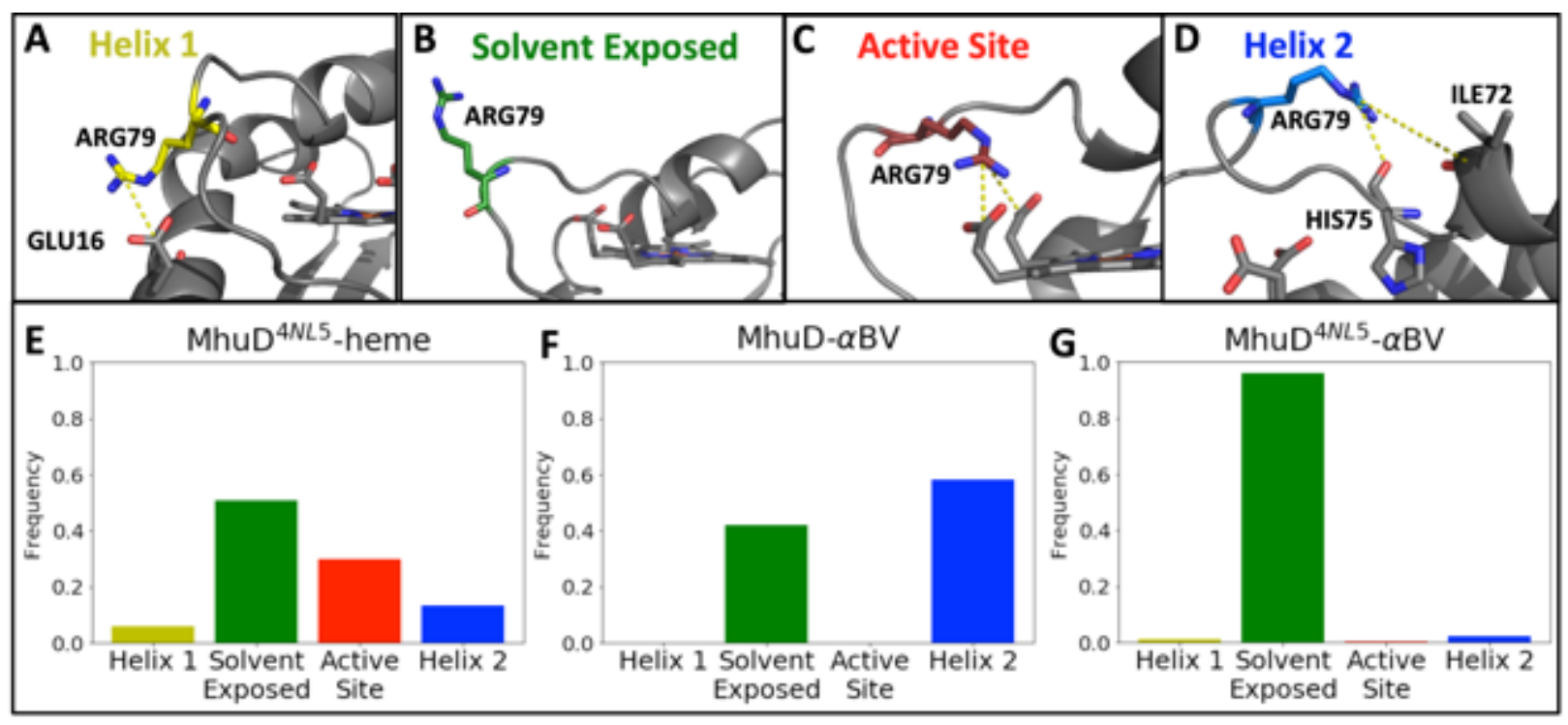




\section{Figure 8}

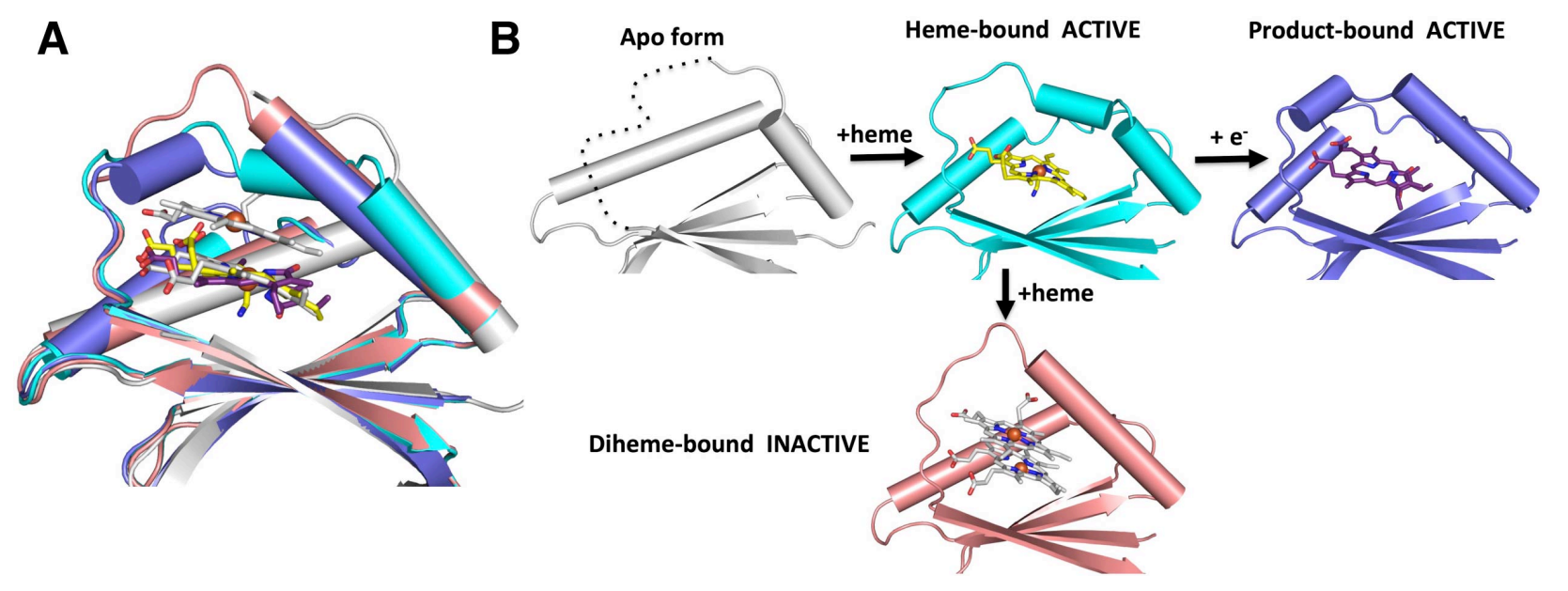




\section{Figure 9}

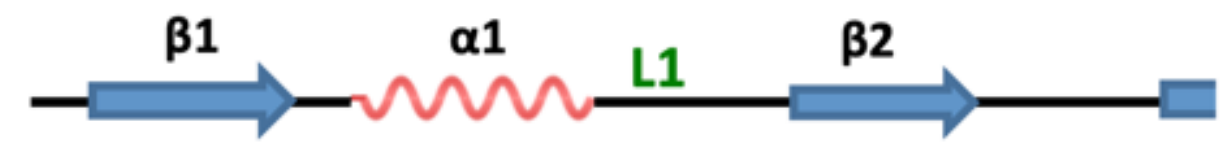

MhuD

--PVVKINAIEVPAGAGPELEKRFAHRAHAVENSPGFLGFQLLRPVKGE--ERYFV

IsdI AHMFMAENRLQLQKGSAEETIERFYNR-QGIETIEGFQQMFVTKTLNTEDTDEVKI

IsdG TMKFMAENRLTLTKGTAKDI IERFYTR-HGIETLEGFDGMFVTQTLEQEDFDEVKI

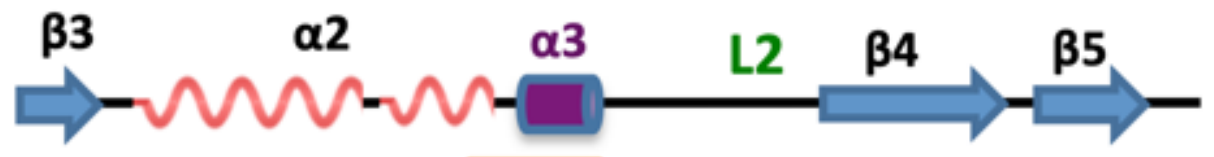

MhuD VTHWESDEAFQAWANGPAI-A HAGHR $\mathrm{N}$ -PVATGASLLEFEVVLDVGGTG

IsdI LTIWESEDSFNNWLNSDVFKE AHKNVRI KSDDDGQQSPILSNKVFKYDIGYHY-QK

IsdG LTVWKSKQAFTDWLKSDVFKA HKHVRS KNEDE--SSPIINNKVITYDIGYSY-MK 